\title{
Study of Plasma-Induced Surface Active Oxygen on Zeolite-Supported Silver Nanoparticles
}

\author{
Yoshiyuki Teramoto $\cdot$ Hyun-Ha Kim • \\ Atsushi Ogata $\cdot$ Nobuaki Negishi
}

Received: 7 May 2013/Accepted: 2 September 2013/Published online: 25 September 2013

(C) The Author(s) 2013. This article is published with open access at Springerlink.com

\begin{abstract}
Surface oxygen induced by non-thermal plasma at atmospheric pressure on silver nanoparticle-loaded zeolite was determined by a chemical probe based on the oxidation of NO. The amount of active oxygen fixed onto the catalyst surface by $\mathrm{O}_{2}$ plasma was approximately proportional to the square of the amount of supported silver. In dry air, its extraordinary long lifetime was confirmed for the first time.
\end{abstract}

Keywords Plasma-driven catalysis - Active oxygen .

Silver nanoparticle $\cdot$ Atmospheric pressure

\section{Introduction}

Environmental issues and especially atmospheric pollution are of great concern throughout the world. Atmosphericpressure non-thermal plasma (NTP) offers unique chemical environment that promotes the removal of air pollutants at low concentrations. On the other hand, the results of studies over the last 30 years have revealed several limitations of plasma-alone processes for practical use in industry. These include large energy consumption, low selectivity, and formation of unwanted byproducts. Plasmacatalyst hybrid technique is a promising technique to overcome these problems.

Combinations of atmospheric pressure dielectric barrier discharge (DBD) with different types of catalyst are currently being studied to improve the process performance

Y. Teramoto $(\bowtie) \cdot$ H.-H. Kim $\cdot$ A. Ogata $\cdot$ N. Negishi

National Institute of Advanced Industrial Science and

Technology (AIST), AIST Tsukuba West, 16-1 Onogawa,

Tsukuba, Ibaraki 305-8569, Japan

e-mail: yoshiyuki-teramoto@aist.go.jp
[1-3]. These combinations have been experimentally proved to provide better selectivity for plasma and lower working temperature for catalyst [4-6]. For example, it has been reported that the combination of DBD and manganese catalyst enhances TCE decomposition and $\mathrm{CO}_{2}$ selectivity $[7,8]$. The loading of active metals not only enhances $\mathrm{CO}_{2}$ selectivity for various VOCs [9-11], but also extends plasma area over the surface on catalyst [12].

The mechanism of synergistic effect, however, is still barely understood. Roland et al. [13] experimentally studied the stabilization of plasma-formed oxidants on the surface of $\mathrm{LaCoO}_{3}$. When $\mathrm{CO} / \mathrm{N}_{2}$ mixture was fed to the $\mathrm{O}_{2}$ plasma pretreated $\mathrm{LaCoO}_{3}$ catalyst, the temporal profiles of outlet $\mathrm{CO}$ concentration changed according to the $\mathrm{O}_{2}$ plasma pretreatment time. They concluded that the active oxygen formed by plasma treatment on the catalyst surface react with $\mathrm{CO}$ to produce $\mathrm{CO}_{2}$ and thus improves the $\mathrm{CO}_{2}$ selectivity, and that the active oxygen is the predominant active species for pollutant oxidation. The same group reported that oxygen atoms in the gas phase are available inside the nano-scale pores of $\gamma-\mathrm{Al}_{2} \mathrm{O}_{3}$ and silica gel, and porosity and specific surface of catalysts are therefore important for efficient pollutant decomposition [1]. Guaitella et al. [14] have shown that the plasma-pretreated $\mathrm{TiO}_{2}$ in $\mathrm{O}_{2}$ or air can remove $\mathrm{C}_{2} \mathrm{H}_{2}$ even in the absence of plasma and UV activation. They predicted that weakly bonded oxygen atoms may remain on $\mathrm{TiO}_{2}$ long after plasma exposure and these react with $\mathrm{C}_{2} \mathrm{H}_{2}$. The adsorbed oxygen species were considered to play a key role in those experiments. More recently, our group reported isotopic evidence on NTP-induced fixation of gas-phase oxygen on the surface of several catalysts such as $\mathrm{TiO}_{2}, \mathrm{Ag} / \mathrm{TiO}{ }_{2}, \mathrm{Ag} /$ $\gamma-\mathrm{Al}_{2} \mathrm{O}_{3}$ and $\mathrm{Ag} / \mathrm{MS}-13 \mathrm{X}$ at atmospheric pressure [15]. Online mass spectrometric analysis and stoichiometric comparison of reactants and products revealed that the fixed 
surface oxygen can be activated by NTP, and it survived for a certain period of time (about $30 \mathrm{~min}$ ).

The object of this letter is to investigate the effect of silver nanoparticles supported on zeolite for plasmainduced active oxygen on the catalyst surface. For this purpose, we quantitatively analyzed, by NO titration, the active oxygen species that was fixed by non-thermal $\mathrm{O}_{2}$ plasma at atmospheric pressure onto the surface of zeolitesupported silver nanoparticles.

\section{Experimental}

The pellet-type zeolite (MS-13X, Sigma-Aldirch Co.) was used in this work. It had a diameter of about $1.6 \mathrm{~mm}$, length of about $2.0-3.0 \mathrm{~mm}$, and specific surface of $475 \mathrm{~m}^{2} / \mathrm{g}$. Silver nanoparticles were supported on the zeolite surface, at loadings of 5, 10, and $15 \mathrm{wt} \%$, by an impregnation method using $\mathrm{AgNO}_{3}$ as a precursor. After several drying processes, the catalysts were calcined at 500 ${ }^{\circ} \mathrm{C}$ in air for $10 \mathrm{~h}$. Figure 1a, b show a TEM image of zeolite with $15 \mathrm{wt} \%$ of silver and diameter distribution of supported silver, respectively. The forms of supported silver nanoparticles were identified as hemispheres ca. 1-3 $\mathrm{nm}$ in diameter.

Figure 2 shows surface dielectric barrier discharge reactor and experimental setup. $1.5 \mathrm{~g}$ of catalyst was set in the effective region of plasma generated by a surface dielectric barrier discharge. The catalyst was held by silicon cap on either side. A stainless steel wire coil was set in contact with the inner wall of a quartz tube (o.d. $=12.5 \mathrm{~mm}$, i.d. $=$ $10.4 \mathrm{~mm}$ ) as a barrier, and silver paste was painted on the outer side of the tube as the ground electrode. The effective length was $30 \mathrm{~mm}$. A high-voltage amplifier (Model 20/20B, Trek Japan K.K., Japan) was used to apply high-voltage ac $\left(100 \mathrm{~Hz}, 28 \mathrm{kV}_{\mathrm{pp}}\right)$ between the electrodes. The discharge power was $45 \mathrm{~J} / \mathrm{L}$ as calculated by the V-Q method. Applied high-voltage was measured with a 1000:1 high-voltage probe (P6015A, Tektronix Corp., USA). The signals of voltage $\mathrm{V}$ and charge $\mathrm{Q}$ were recorded with a digital oscilloscope (Tektronix, TDS3034B Corp., USA). The area of
V-Q parallelogram was calculated using an automated V-Q Lissajous program (V-Q Lissajous Program ver. 1.71, Insight Inc. Corp., Japan). The gas flow rate was fixed at $1.0 \mathrm{~L} / \mathrm{min}$ using mass flow controllers (KOFLOC, FCC3000). All experiments were conducted under dry conditions. Concentration of water vapor was less than about $80 \mathrm{ppmv}$, as measured by a dew point hygrometer (General Eastern, Hygro-M4 Model).

The active oxygen fixed by $\mathrm{O}_{2}$ plasma pretreatment on catalyst surface was measured with NO oxidation method. The experimental procedure consists of several steps. First the catalysts were pretreated for $20 \mathrm{~min}$ under the conditions listed in Table 1. Then $\mathrm{N}_{2}$ gas was supplied to remove all the gas phase species generated by plasma in the reactor. Afterwards the mixture of $100 \mathrm{ppm} \mathrm{NO}$ in $\mathrm{N}_{2}$ was introduced and the outlet $\mathrm{NO}$ concentration was monitored by an on-line FT-IR spectrometer (Perkin-Elmer, Spectrum One) at $15 \mathrm{~s}$ intervals. The examples of FT-IR spectra were shown in Fig. 3. The experiment was repeated 30 times for each condition and the average values were plotted in Fig. 4. The gas temperature in reactor during plasma pretreatment was about $48^{\circ}$ measured by fiber optic

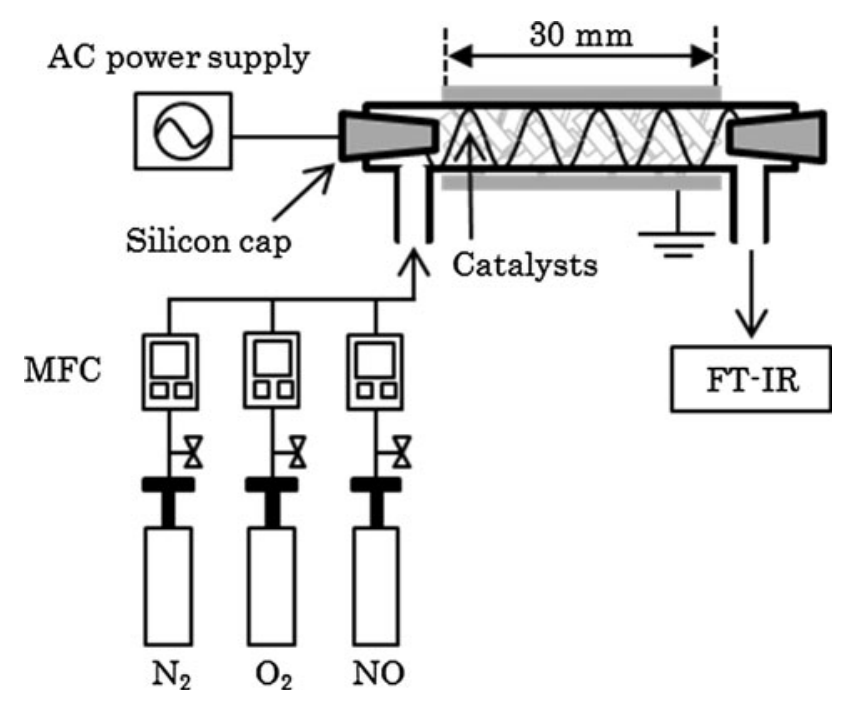

Fig. 2 Experimental setup
Fig. 1 a TEM image of $\mathrm{Ag}$ $15.0 \mathrm{wt} \% / \mathrm{MS}-13 \mathrm{X}, \mathbf{b}$ diameter distribution of supported silver nanoparticles
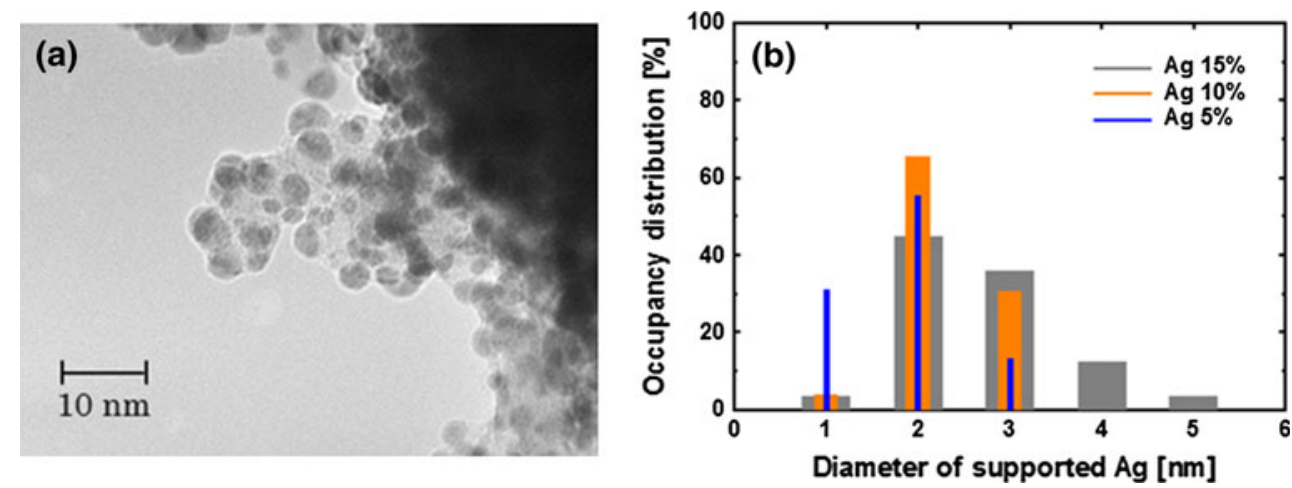
Table 1 Pretreatment condition

\begin{tabular}{llll}
\hline Condition & Ag amount on MS-13X & Plasma & Gas \\
\hline a & No catalyst & $\times$ & $\mathrm{O}_{2}$ \\
$\mathrm{~b}$ & $15 \mathrm{wt} \%$ & $\times$ & $\mathrm{O}_{2}$ \\
c & $0 \mathrm{wt} \%$ & $\bigcirc$ & $\mathrm{O}_{2}$ \\
d & $5 \mathrm{wt} \%$ & $\bigcirc$ & $\mathrm{O}_{2}$ \\
e & $10 \mathrm{wt} \%$ & $\bigcirc$ & $\mathrm{O}_{2}$ \\
$\mathrm{f}$ & $15 \mathrm{wt} \%$ & $\bigcirc$ & $\mathrm{O}_{2}$ \\
g & $15 \mathrm{wt} \%$ & $\bigcirc$ & $\mathrm{N}_{2}$ \\
\hline $\mathrm{O}$ and $\times$ show with and without plasma treatment, respectively
\end{tabular}
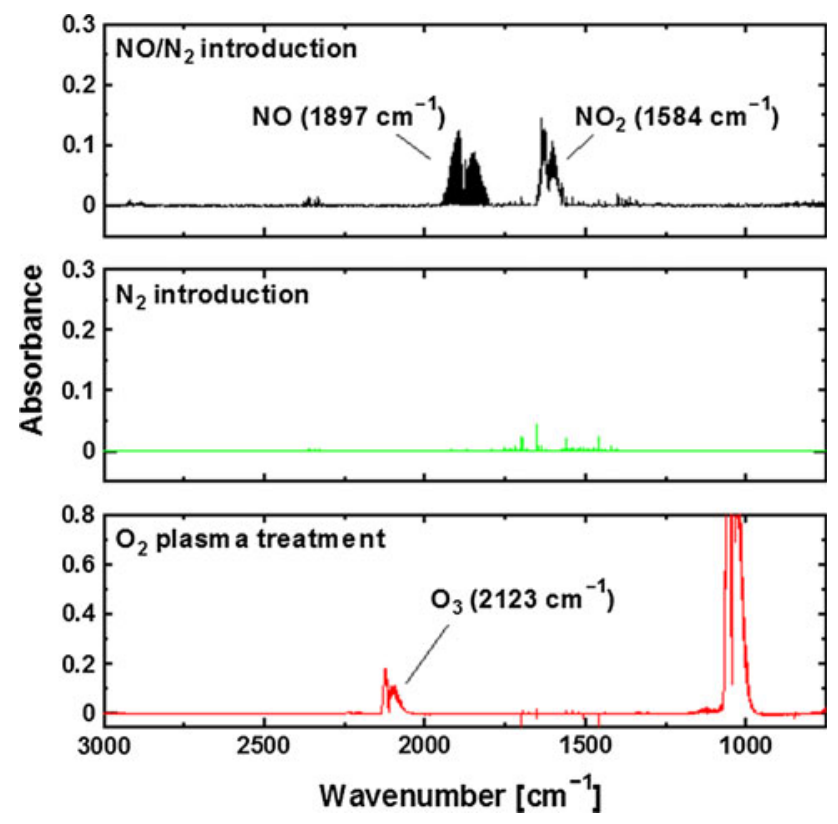

Fig. 3 FT-IR spectra

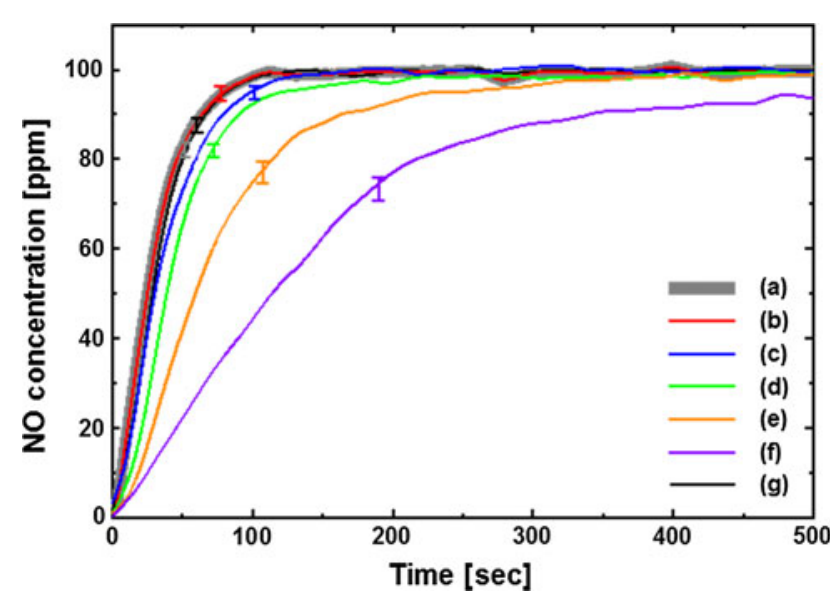

Fig. 4 Temporal profiles of NO concentration with catalysts pretreated under conditions described in Table 1 thermometer (AMOTH FL-2000, ANRITSU), and it was stable between start and end.

\section{Results and Discussions}

Figure 4 shows temporal profiles of outlet NO concentrations after the introduction of $\mathrm{NO}(100 \mathrm{ppm}) / \mathrm{N}_{2}$. The letters (a-g) assigned to the curves in Fig. 4 correspond to the conditions described in Table 1. Adsorption of NO on the tested catalysts was found to be negligible, since the temporal profiles were identical regardless of whether catalyst was present, as can be seen in (a) and (b) in Fig. 4. This observation validates the NO titration method for measuring the plasma-induced surface oxygen. The small shift in the rise time induced by $\mathrm{O}_{2}$ plasma pretreatment for silverunloaded zeolite (compare (a) and (c) in Fig. 4), could be due to NO oxidation reaction with fixed active oxygen on zeolite surface or with $\mathrm{O}_{3}$ retained by zeolite. In either case, these were predicted to be low amount from the small shift.

On the other hand, noticeable changes of profiles $(\mathrm{d}, \mathrm{e}, \mathrm{f})$ were observed with the silver-loaded zeolite pretreated by $\mathrm{O}_{2}$ plasma. The surface active oxygen fixed by $\mathrm{O}_{2}$ plasma pretreatment increased with increasing amount of supported silver and it is assumed that the concentration of NO decreased by its oxidation reaction with active oxygen on the catalyst surface. In other words, the Ag nanoparticles played the key role in the fixation of oxygen species, which in turn led to the enhanced reactivity. However, there was a possibility that plasma created (i) new adsorption sites or (ii) active sites such as lattice defects on catalyst surface. To find out the influence of these two factors on NO reduction, the $\mathrm{N}_{2}$ plasma pretreatment was applied to silver-loaded zeolite. In this case, a decrease in NO concentration was not observed (Fig. 4g). It indicates that plasma pretreatment is unable to create new adsorption sites and active sites on catalyst surface; thus, influence on NO reduction by (i) and (ii) can be ruled out in this experiment. Moreover, rapid rise of $\mathrm{NO}_{2}$ concentration was detected at the time of NO introduction for silver-loaded zeolite pretreated by $\mathrm{O}_{2}$ plasma. This allows us to conclude that some active oxygen fixed by $\mathrm{O}_{2}$ plasma pretreatment oxidizes $\mathrm{NO}$ to $\mathrm{NO}_{2}$. Such oxidizing ability was also confirmed by $\mathrm{CO}$ titration and $\mathrm{CO}_{2}$ detection (data not shown).

Although the amount of active oxygen fixed by plasma on catalysts can be estimated indirectly from the amount of $\mathrm{NO}_{2}$ emission, this method is subject to large errors due to a high adsorption capability of zeolite for $\mathrm{NO}_{2}$. Therefore, the amount of active oxygen was estimated from the amount of removed NO which is integrated value of the differences of NO concentration between (a) and (c-f) in Fig. 4. For all the different loadings of silver, the amount 


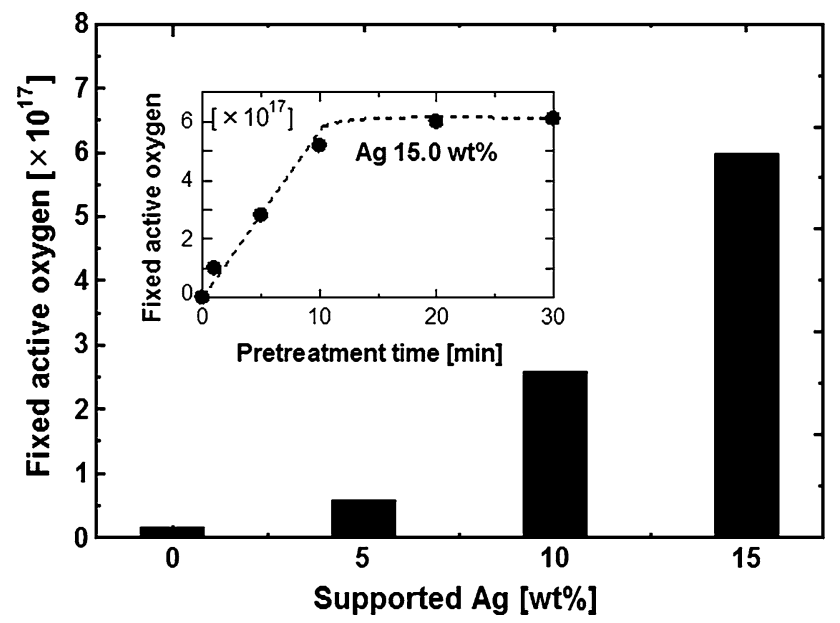

Fig. 5 Amount of plasma-induced surface oxygen species as a function of the amount of supported silver. Inset shows amount of fixed active oxygen as a function of pretreatment time

of fixed active oxygen was proportional to the pretreatment time up to $10 \mathrm{~min}$ and then leveled off (an example is shown in inset of Fig. 5). Furthermore, the final amounts of fixed active oxygen, which are shown in Fig. 5, were proportional to the square of the amounts of supported silver. This effect can be explained by extension of plasma area over the catalyst surface with the increasing amount of supported silver nanoparticles.

The surface density of active oxygen on silver nanoparticles was roughly calculated from the data in Figs. 1 and 5. Based on the assumption that supported silver particles were hemispherical with diameter of $2 \mathrm{~nm}$, the total surface area of silver at the loading of $15 \mathrm{wt} \%$ was estimated to be $60.1 \mathrm{~m}^{2}$, and the surface density of active oxygen on catalyst was calculated to be $1.0 \times 10^{12} \mathrm{~cm}^{-2}$. Roland et al. [13] have reported that the surface density of active oxygen fixed by $\mathrm{O}_{2}$ plasma pretreatment on $\mathrm{LaCoO}_{3}$ was estimated to be about $1-2.5 \times 10^{12} \mathrm{~cm}^{-2}$ using $\mathrm{CO}$ oxidation reaction. The comparison of the surface density of active oxygen between $\mathrm{LaCoO}_{3}$ and zeolite-supported silver nanoparticles indicates that both materials have similar capabilities of oxygen storage during $\mathrm{O}_{2}$ plasma.

The decay of fixed active oxygen was studied in different gas environments using the catalyst pretreated under the condition denoted as (f) in Table 1. Following plasma pretreatment, $\mathrm{N}_{2}$ or dry air was supplied during $1,10,100$, and $1000 \mathrm{~min}$. Afterwards the residual active oxygen on catalyst was determined by same method as previously described.

The lifetimes of surface-fixed oxygen in $\mathrm{N}_{2}$ or dry air are shown on the Fig. 6. More than 90 and $70 \%$ of initial amount of active oxygen survived for $1000 \mathrm{~min}$ in $\mathrm{N}_{2}$ and dry air atmosphere, respectively. By contrast, in dry air the

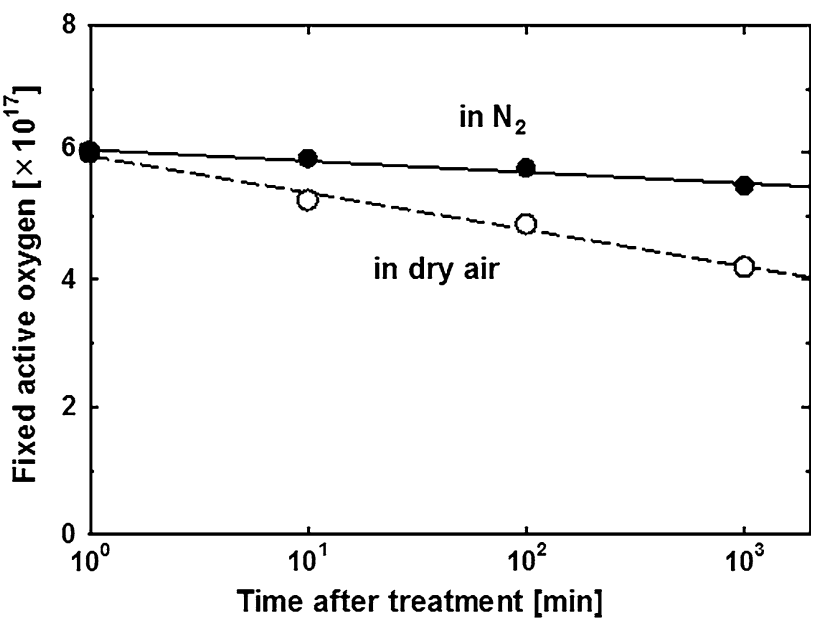

Fig. 6 Lifetime of plasma-induced surface oxygen species in $\mathrm{N}_{2}$ and in dry air

gas phase highly reactive oxygen species, atomic oxygen, electron-excited oxygen etc., decreased to $10 \%$ or less by the three-body reaction within $100 \mu$ s after the discharge [16]. Such short lifetimes are one of the factors in performance limitations of the plasma-alone processes. The interaction of plasma with catalyst can fix gas-phase reactive oxygen on the surface of silver-loaded zeolite, and significantly extend the lifetime of fixed active oxygen.

\section{Summary}

In the present study, plasma-induced surface active oxygen on silver nanoparticles-loaded zeolite has been investigated at atmospheric-pressure using NO titration. The amount of active oxygen fixed by $\mathrm{O}_{2}$ plasma on catalyst surface was approximately proportional to the square of the amount of supported silver, and it was proportional to pretreatment time up to $10 \mathrm{~min}$ and then leveled off. Additionally, in $\mathrm{N}_{2}$ and dry air extraordinary long lifetime of fixed active oxygen was confirmed for the first time. These results indicate that supported silver nanoparticles on zeolite play significant role for performance improvement in plasmacatalyst hybrid technique, and proposed an explanation for the synergy in plasma-catalyst hybrid process.

In future work, identification of plasma-induced surface active oxygen species are required for detailed understanding of reaction mechanisms on plasma-catalyst hybrid process.

Open Access This article is distributed under the terms of the Creative Commons Attribution License which permits any use, distribution, and reproduction in any medium, provided the original author(s) and the source are credited. 


\section{References}

1. Holzer F, Roland U, Kopinke FD (2002) Appl Catal B 38:163

2. Guaitella O, Thevenet F, Puzenat E, Guillard C, Rousseau A (2008) Appl Catal B 80:296

3. Kim HH, Ogata A, Futamura S (2005) J Phys D Appl Phys 38:1292

4. Kim HH (2004) Plasma Process Polym 1:91

5. Chen HL, Lee HM, Chen SH, Chang MB, Li SN (2009) Environ SciTechnol 43:2216

6. Sano T, Negishi N, Sakai E, Matsuzawa S (2006) J Mol Catal A 245:235

7. Magureanu M, Mandache NB, Parvulescu VI, Subrahmanyam C, Renken A, Kiwi-Minsker L (2007) Appl Catal B Environ 74:270

8. Han SB, Oda T, Ono R (2005) IEEE Trans Ind Appl 41:1343
9. Kim HH, Oh SM, Ogata A, Futamura S (2004) Catal Lett 96:189

10. Hakoda T, Matsumoto K, Mizuno A, Kojima T, Hirota K (2008) Plasma Chem Plasma Process 28:25

11. Rousseau A, Meshchanov AV, Roepcke J (2006) Appl Phys Lett 88:021503

12. Kim HH, Kim JH, Ogata A (2009) J Phys D Appl Phys 42:135210

13. Roland U, Holzer F, Kopinke FD (2002) Catal Today 73:315

14. Guaitella O, Lazzaroni C, Marinov D, Rousseau A (2010) Appl Phys Lett 97:011502

15. Kim HH, Ogata A, Schiorlin M, Marotta E, Paradisi C (2011) Catal Lett 141:277

16. Ono R, Yamashita Y, Takezawa K, Oda T (2005) J Phys D Appl Phys 38:2812 